\title{
Air parcel random walk and droplet spectra broadening in clouds
}

\author{
K.S. Turitsyn* \\ Physics of Complex Systems, Weizmann Institute of Science, Rehovot 76100, Israel
}

\begin{abstract}
We study the effect of turbulent flow on the droplet growth in a cloud during the condensation phase. Using the air parcel model, we describe analytically how the size distribution of droplets evolves at the different stages of parcel movement. We show that turbulent random walk superimposed on an accelerated ascent of the parcel makes the relative width of droplet distribution to grow initially as $t^{1 / 2}$ and then decay as $t^{-3 / 2}$.
\end{abstract}

PACS numbers: 05.40.-a, 47.27.-i

The growth mechanism of small water droplets in clouds is the condensation of water vapour [1]. While the dynamics of droplet growth is well understood, no consistent statistical theory exists yet for describing the evolution of droplet spectra in the cloud - see [1, 2] and the references therein. The main difficulties in this task are that a consistent theory should describe the interaction of at least four fields in the cloud: vapour concentration, temperature, droplet distribution and velocity. For sufficiently small droplets, the concentration inhomogeneities due to droplet inertia are negligible [3, 4, 5]. In cloud cores, vapour concentration and temperature also can be considered smooth functions of position, so here we shall only consider their vertical dependence. It is the velocity field which has a turbulent nature and can be considered as a stochastic variable. The statistical description of random interacting fields belongs to the class of field-theoretical problems which are generally unsolvable. In our case, neglecting the spatial fluctuations of concentrations and temperature, one can turn the problem into that of a random walk superimposed on a regular drift in the prescribed environment. Three limiting cases of that problem (with no drift, permanent-velocity drift and permanent-acceleration drift) are solved here analytically. This might provide a useful opportunity for comparison with the data of observations and numerical simulations. We use the concept of an air parcel [1], which means that we consider a macroscopic volume of air, which is moving through the cloud with the droplets within it. Together with the deterministic motion and growth, the parcel participates in a turbulent random walk which can be modelled as brownian motion on a timescale larger than the velocity correlation time. We examine how the form of the droplet size distribution changes with time. It is shown that the dynamics of the form of the distribution (spectrum) strongly depends on the deterministic motion. The relative spectral width decreases while the parcel is moving upwards either with constant acceleration or with a constant velocity. When there is no vertical drift (the parcel comes into mechanical equilibrium with its environment and wanders around a fixed height), the relative width of the distribution begins to grow. The form of the spectrum increases in width. We also show, that the distribution of droplets over sizes is asymmetric: power law at small sizes part is determined by the activation of new nuclei while the large sizes

feturits@wicc.weizmann.ac.il part is exponential.

Advection and diffusion of passive fields in turbulent fluid is usually described with the Kraichnan model (see e.g. [6]). According to this model the velocity field can be considered a stochastic Markovian process. The stochastic description is valid when the fluid motion is chaotic. This is true in the case of fully developed turbulence which corresponds to large values of Reynolds number [7]. In real clouds the observed Reynolds number is of order $\mathrm{R} e \sim 10^{5} \div 10^{8}$ [1], which is certainly enough to assume air velocity field stochastic. The validity of the zero correlation time assumption is proved by the following arguments: correlation times of velocity fluctuations do not exceed $10^{2} \mathrm{~s}$, while the duration of the condensational stage lasts $10^{3} \mathrm{~s}$ or more [1]. Thus a more realistic model, which takes into account the finite correlation times would give a small correction not exceeding $10 \%$. In this paper we focus only on the vertical motion of a particle, because horizontal inhomogeneities have fluctuating nature, and will not lead to any new qualitative effect. We also neglect collisions and coalescence of droplets by considering the initial stage of growth when droplets are small and the only relevant droplet growth mechanism is condensation of water vapour[1]. Using all these assumptions, one can write the set of equations describing the dynamics of a single droplet [1, 2]:

$$
\begin{aligned}
& \frac{\mathrm{d} A}{\mathrm{~d} t}=\frac{\mathrm{d} a^{2}}{\mathrm{~d} t}=s(z, t), \\
& \frac{\mathrm{d} z}{\mathrm{~d} t}=u(t)+v(t) .
\end{aligned}
$$

Here $a$ is the droplet radius, $A=a^{2}$ is a variable, proportional to the droplet surface area, and $s$ is the local supersaturation rate defined as $s=n / n_{0}-1$, where $n$ and $n_{0}$ are the local vapour concentration and the concentration of saturated vapour correspondingly. We have set the coefficient of propotionality to 1 , which can be always achieved by using the proper dimensional units. For any given droplet, the supersaturation rate changes with time because the air parcel containing this droplet is lifted up to the cloud and is being cooled along the way. The vertical velocity of a parcel (2) consists of two components: the deterministic $u(t)$, which corresponds to the macroscopic updraft flow and the random $v(t)$ which is due to turbulent velocity fluctuations. We will consider the random velocity to be gaussian and short correlated

$$
\left\langle v(t) v\left(t^{\prime}\right)\right\rangle=D \delta\left(t-t^{\prime}\right) .
$$

In the following we will analyze the dynamics of the droplet 

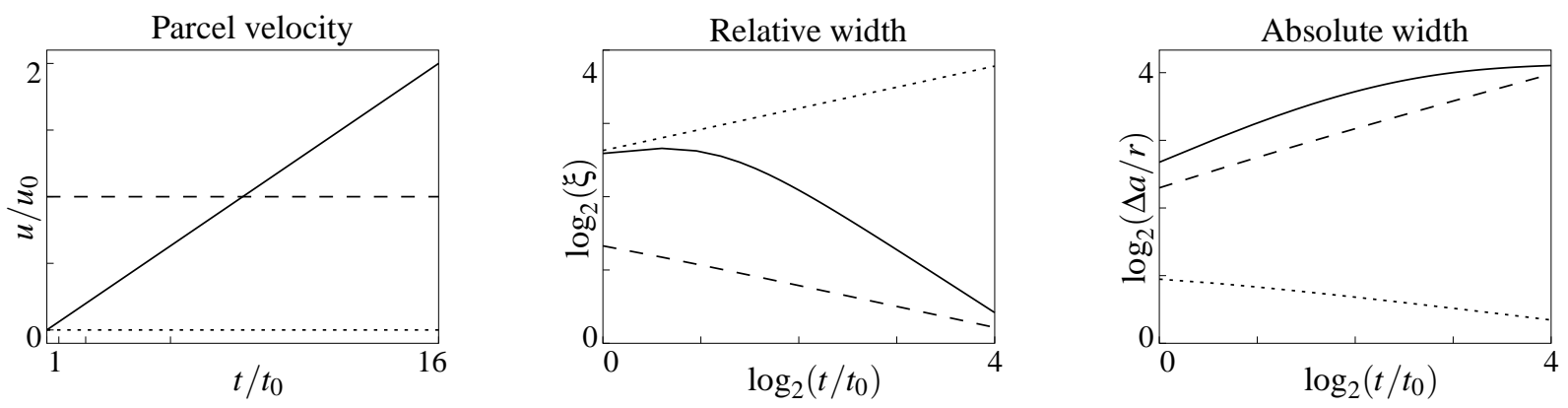

FIG. 1: An example of the parcel deterministic velocity trajectory together with the relative and absolute distribution width for arbitraty chosen parameters $t_{0}, u_{0}, r$. The three curves in each plot correspond to the different cases: parcel with constant acceleration (solid line), parcel with constant velocity (dashed line) and parcel with zero velocity (dotted line).

distribution $P(A, z, t)$. The quantity $P(A, z, t) \mathrm{d} A \mathrm{~d} z$ represents the fraction of the droplets which are located in the area $(z, z+\mathrm{d} z)$ and have the surface area in the interval $(A, A+\mathrm{d} A)$. As long as we do not take into account the interaction of the droplets, the dynamical equation governing the evolution of the spectrum is described by the Liouville equation that corresponds to the single droplet dynamics (112):

$$
\left\{\partial_{t}+s(z, t) \partial_{A}+[u(t)+v(t)] \partial_{z}\right\} P(A, z, t)=0 .
$$

Applying the standard procedure of averaging over the white noise (3) one obtains the Fokker-Planck equation:

$$
\left\{\partial_{t}+s(z, t) \partial_{A}+u(t) \partial_{z}-\frac{1}{2} D \partial_{z}^{2}\right\} P(A, z, t)=0 .
$$

The second and third terms describe the advection of the droplets in phase space due to deterministic terms in equations (12). They are responsible for the motion of the distribution center. The third term comes from the stochastic part of the velocity and its main effect is the spectrum broadening. We assume that the initial droplet size is small so that we can set $P(A, z, 0)=\delta(A) \delta(z)$. This equation can not be solved for general supersaturation field $s(z, t)$. However it has an analytic solution for a stationary linear profile $s(z, t)=s_{0}+s_{1} z$ which we consider as a reasonable local approximation of the real supersaturation field. The expression for $P(A, z, t)$ is rather bulky. However the size distribution $P(A, t)=\int \mathrm{d} z P(A, z, t)$ is described by the simple expression

$$
P(A, t)=\frac{1}{\sqrt{2 \pi \delta A^{2}(t)}} \exp \left[-\frac{\left(A-A_{0}(t)\right)^{2}}{2 \delta A^{2}(t)}\right] .
$$

Here $A_{0}(t)=\int_{0}^{t} \mathrm{~d} t^{\prime}\left(s_{0}+s_{1} z_{p}\left(t^{\prime}\right)\right)$ and $z_{p}(t)$ is a deterministic parcel trajectory: $z_{p}(t)=\int_{0}^{t} \mathrm{~d} t^{\prime} u\left(t^{\prime}\right)$. The distribution width is $\delta A^{2}(t)=D s_{1}^{2} t^{3} / 3$. Usually one is interested in the distribution of radius $a$; from (6) we see that it will be substantially nongaussian in our case as $P_{a}(a, t)=2 a P\left(a^{2}, t\right)$. Nevertheless, it will still have the bell-form and can be described by it's center $a_{0}(t)=\sqrt{A_{0}}$ and width

$$
\delta a(t)=\sqrt{A_{0}+\delta A}-\sqrt{A_{0}}=\frac{\delta A}{\sqrt{A_{0}+\delta A}+\sqrt{A_{0}}} .
$$

One can see that in some situations the distribution in $a$ can be narrowed, even if it is broadened for the variable $A$. The growth rate of the distribution width can be described by the variable $\xi=\delta A / A_{0}$. The relative width of the distribution over radii is then

$$
\frac{\delta a}{a_{0}}=\frac{\xi}{\sqrt{1+\xi}+1}
$$

The r.h.s. of this relation is a monotonic function, so the relative width of the distribution over $a$ behaves qualitatively in the same way as the variable $\xi$. Even the regular (nonturbulent) part of the parcel motion in a real cloud can be very complicated. At the beginning the parcel is being lifted up the cloud because of the convection mechanism. The parcel is accelerated until the velocity reaches some stationary value due to the drag force. During the motion the temperature of the parcel changes because of droplet growth and heat exchange. When the parcel temperature becomes equal to the ambient temperature, its velocity decreases to zero and it stays at this steady height. One can analyze such a movement by considering three limiting cases: when the parcel is accelerated so $u(t)=u_{0}+\mathbf{a} t ;$ when the parcel's velocity is constant: $u(t)=u$; and when the parcel has reached its steady height, and its velocity is zero, $u(t)=0$. In the following sections we will analyze these three cases and show that they correspond to the different types of behaviour of the size distribution function.

We start from the simplest case of zero velocity. As already mentioned, this case is important when analyzing the last part of the parcel's movement. On this part the distribution center has already moved from $A=0$ and its width is also finite. However if the steady stage lasts for a long time, the initial conditions are forgotten, and one can treat this problem with the initial conditions $A(0)=0$ and $\delta A(0)=0$. The solution of this problem was introduced in the previous section (6), and one obtains: $z_{p}(t)=0, A_{0}(t)=s_{0} t, \delta A^{2}(t)=D s_{1}^{2} t^{3} / 3$. Thus for the relative width one finds:

$$
\xi=\frac{\delta A}{A}=\sqrt{D / 3} \frac{s_{1}}{s_{0}} t^{1 / 2} .
$$

Therefore the distribution is being broadened with a rate $\xi \propto$ $t^{1 / 2}$ at this stage. Turning to the radius distribution, $P_{a}(a, t)$, one can say that its relative width is increasing with the same rate $\delta a / a \propto \xi \propto t^{1 / 2}$ for small times $t<s_{0}^{2} / s_{1}^{2} D$, but as $\xi$ becomes large enough according to 8 its growth rate changes 
to $\delta a / a \propto \xi^{1 / 2} \propto t^{1 / 4}$. The radius distribution is broadened slower than that of the surface area. It is worth noting that in stratiform clouds where the deterministic parcel movement is small the zero velocity case is most important. We see,that one should observe strongly broadened distributions there.

Next we consider the constant velocity case: $z_{p}(t)=u t$, $A_{0}(t)=A_{0}(0)+s_{0} t+\left(s_{1} u / 2\right) t^{2}$. Again, at large enough times, the distribution forgets about the initial conditions (which were formed during the acceleration stage) and the last term in $A_{0}(t)$ will become dominant, so $A_{0}=\left(s_{1} u / 2\right) t^{2}, \delta A^{2}=$ $\left(D s_{1}^{2} / 3\right) t^{3}$. Therefore the relative distribution width is slowly decreasing: $\xi \propto t^{-1 / 2}$, while the center of the distribution and its absolute width are growing with the rates $a_{0}=\sqrt{A_{0}} \sim t$, $\delta a \sim \sqrt{A_{0}} \xi \propto t^{1 / 2}$.

Finally, we consider a uniformly accelerating parcel. Here one finds $s(t)=s\left(z_{p}(t)\right)=s_{0}+s_{1} u_{0} t+s_{1} \mathbf{a} t^{2}$. If initial droplets sizes are small, we can easily find the dynamics of the center of drops surface area distribution, $A_{0}(t)=s_{0} t+\left(s_{1} u_{0} / 2\right) t^{2}+$ $\left(s_{1} \mathbf{a} / 6\right) t^{3}$. The relative width behaves like

$$
\xi=\frac{\delta A}{A_{0}}=\frac{\sqrt{D s_{1}^{2} / 3} t^{3 / 2}}{s_{0} t+\left(s_{1} u_{0} / 2\right) t^{2}+\left(s_{1} \mathbf{a} / 6\right) t^{3}} .
$$

At the very small times the relative width is growing with the rate $\xi \propto t^{1 / 2}$, as in the zero velocity case. At large times, when the parcel has accelerated enough, the relative width tends to zero as $\xi \propto t^{-3 / 2}$. Therefore, there is a time when the relative width has a maximum. The position of the maximum is determined by the values of $u_{0}, \mathbf{a}$. It can be estimated as $t_{\max } \sim \min \left[s_{0} /\left(s_{1} u_{0}\right), \sqrt{s_{0} /\left(s_{1} \mathbf{a}\right)}\right]$. The value of the maximum is in this case $\xi_{\max } \sim \sqrt{D t_{\max }} s_{1} / s_{0}$. It is interesting, that the absolute width $\delta a=\sqrt{A_{0}}(\sqrt{1+\xi}-1) \sim \sqrt{A_{0}} \xi$ is always increasing, and approaches a constant asymptotic value at large times. The particular value of this width is a complicated function of all the parameters.

The figure 1 shows the qualitative behaviour of the absolute and relative width for the three analyzed cases. The pictures should not be considered as a prediction for some particular cloud, but as an example, which shows how different velocity trajectory can affect the distribution parameters.

So far we have analyzed the situation where small droplets are injected into the cloud only once, at the beginning. In real clouds the situation is certainly different. New nuclei are activated all the time, and their activation surely affects the distribution form. Nevertheless, we will show that this effect of new nuclei activation does not change the large droplet sizes part of the distribution. The droplet size distribution can be written as follows:

$$
n(a, t)=\int_{0}^{t} \mathrm{~d} t^{\prime} n_{0}\left(t^{\prime}\right) P_{a}\left(a, t-t^{\prime}\right),
$$

where $n_{0}(t) \mathrm{d} t$ is the number of nuclei activated in the interval $(t, t+\mathrm{d} t)$. Luckily the theory of nuclei activation is well developed [1]. The simplest model of nuclei activation predicts, that the activation rate is proportional to some power of local supersaturation rate, $n_{0}(t) \propto s^{-k}(t)$, and usually $k>0$. The absolute width $\delta a(t)$ of the distribution $P_{a}(a, t)$ is a growing function, therefore the front of the real distribution will be always determined by the droplets which began to grow at $t=0$. Nevertheless the new droplets determine the form of the left part. Assuming the distribution to be narrow enough, one can have the following approximation to the small droplet part of the spectrum: $n\left(a_{0}-\Delta a, t\right) \sim n_{0}\left(t^{*}\right)$, where $t^{*}$ is determined by the condition that $P_{a}\left(a, t^{*}\right)$ should be centered at $a-\Delta a$. For example for the acceleration stage $s(t)=s_{0}+s_{1} \mathbf{a} t^{2} / 2$, and the center position is given by $A_{0}(t)=s_{0} t+s_{1} \mathbf{a} t^{3} / 6 \propto$ $t^{3}$. Thus $a_{0}(t) \propto t^{3 / 2}$. From the definition of $t^{*}$ we find $t-t^{*} \propto\left(a_{0}-\Delta a\right)^{2 / 3}$, so $t^{*} \propto a_{0}^{2 / 3}-\left(a_{0}-\Delta a\right)^{2 / 3} \propto a_{0}^{-1 / 3} \Delta a$ for small $\Delta a \ll a_{0}$. Recalling that $n_{0}(t) \propto s^{-k}$, we find that $n\left(a_{0}-\Delta a, t\right) \propto\left(s_{0}+c \Delta a^{2}\right)^{-k}$. We can see that the left part will have a rather wide power law tail distinct from a narrow exponential right tail. This is also an important result, which might be observed in real clouds.

To conclude, we have shown that the evolution of size distribution of droplet spectra can be divided into three different stages. At each stage, the relative width of the distribution behaves according to different power laws: it grows with a rate $\xi \propto t^{1 / 2}$ at the zero velocity stage, and decays with the laws $\xi \propto t^{-1 / 2}, \xi \propto t^{-3 / 2}$ on the stages with parcel constant velocity, constant acceleration respectively. The dynamics of real air parcels is more complicated, and the equation (6) allows one to estimate the distribution width according to our model. However, from physical reasoning we see that real parcel dynamics can be approximated by three consequent stages, where the relative width asymptotics were computed analytically.

We have also shown, that the left part of droplet size spectra is mainly determined by the process of nuclei activation during the initial stage of parcel movement. In this case one will observe a power-law tail in the small droplets part of spectrum, in contrary to exponential tail for large droplet sizes, which is determined by the parcel's velocity fluctuations.

Acknowledgements: Author would like to thank G. Falkovich for the most fruitful and inspiring discussions and M.G. Stepanov for useful remarks and technical assistance in making figures.
[1] H. Pruppacher \& Klett, J. Microphysics of Clouds and Precipitation (Kluwer, Dordrecht 1998).

[2] A. Korolev, J. Atm. Sci. 52, 3620-3634 (1995).
[3] Wang, L. P. \& Maxey, M. Settling velocity and concentration and concentration distribution of heavy particles in homogeneous isotropic turbulence. J. Fluid Mech. 256, 27-68 (1993). 
[4] Squires, K. \& Eaton, J. Measurements of particle dispersion from direct numerical simulations of turbulence. J. Fluid Mech. 226, 1-35 (1991).

[5] G. Falkovich, A. Fouxon and M.G. Stepanov, Acceleration of rain initiation by cloud turbulence, Nature 419, 151-154 (2002)
[6] G. Falkovich, K. Gawedzki and M. Vergassola, Particles and fields in fluid turbulence, Rev. Mod. Phys. 73,913-975 (2001)

[7] U. Frisch, Turbulence. The Legacy of A.N.Kolmogorov (Cambridge University Press, Cambridge, England) (1995) 